\title{
Supercondutividade: cem anos de desafios
}

Paulo Pureur

\section{O DESAFIO DAS BAIXAS TEMPERATURAS}

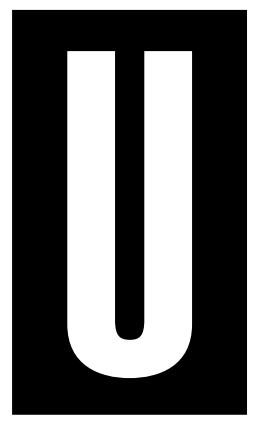

$m$ dos mais espetaculares fenômenos da física dos materiais sólidos é a supercondutividade. Nos sistemas supercondutores, a resistência à passagem de uma corrente elétrica contínua é total e abruptamente suprimida quando o material é resfriado abaixo de uma temperatura bem definida, denominada de temperatura crítica, a qual é usualmente simbolizada como $T_{\text {c }}$.

A resistência elétrica depende do formato da amostra e é proporcional à resistividade elétrica, que é uma propriedade do material.
O valor da resistividade expressa a dificuldade oferecida pelo material à passagem de uma corrente elétrica em seu interior. No caso dos sistemas metálicos, elétrons desvinculados de seus átomos de origem, denominados de elétrons de condução, asseguram o transporte de carga elétrica com muita eficácia. Assim, os metais são bons condutores elétricos e os valores da resistividade elétrica são relativamente baixos nesses sistemas. A Figura 1 ilustra esquematicamente a resistividade do metal prata no regime de temperaturas próximas ao zero absoluto (a temperatura do zero absoluto - zero Kelvin - corresponde aproximadamente a $-273^{\circ}$ Celcius). Nessa região, a resistividade da prata é aproximadamente constante, pois deve-se aos choques dos elétrons de condução com as imperfeições estáticas da estrutura cristalina.

\section{PRÊMIOS NOBEL EM SUPERCONDUTIVIDADE E CONTRIBUIÇÃO}

Heike
Kamerlingh Onnes
Propriedades da
matéria em baixas
temperaturas,
incluindo
descoberta da
supercondutividade
e liquefação do hélio

- Leon N. Cooper
- J. Robert Schrieffer
Desenvolvimento da
teoria microscópica da
supercondutividade,
hoje conhecida
como teoria BCS

\begin{tabular}{c|ccc|} 
& & & \\
- Brian & - Ivar Giaver & \\
D. Josephson & & & \\
Predição teórica & Tunelamento de & Leo Esaki recebeu \\
do tunelamento de & elétrons através & o prêmio em \\
pares de Cooper & de barreira & razão de estudos \\
através de uma fina & isolante entre & sobre tunelamento \\
barreira isolante & supercondutores & quântico em \\
entre eletrodos & semicondutores \\
supercondutores & $\vdots$ & &
\end{tabular}


Em 1911, Heike Kamerlingh Onnes, da Universidade de Leiden, na Holanda, estudava a variação da resistividade do metal mercúrio em temperaturas muito baixas quando observou - pela primeira vez - o fenômeno da supercondutividade ${ }^{1}$. A Figura 1 ilustra o súbito desaparecimento da resistividade desse metal na temperatura crítica $T_{\mathrm{c}}=4,15 \mathrm{~K}$.

A descoberta da supercondutividade foi precedida da superação de um grande desafio tecnológico: a obtenção, em laboratório, de temperaturas próximas ao zero absoluto. De fato, em 1908, Onnes tivera êxito ao liquefazer o gás hélio (He), cujo ponto de ebulição corresponde a $\mathrm{T}=4,2 \mathrm{~K}$ (equivalente a $-269^{\circ} \mathrm{C}$ ). Assim, o He líquido passou a ser usado como reservatório térmico para esfriar materiais a temperaturas muito baixas. Diminuindo-se a pressão de vapor sobre o líquido do isótopo ${ }^{4} \mathrm{He}$, pode-se alcançar temperaturas próximas a $1 \mathrm{~K}$. É interessante ressaltar que, mesmo nos dias presentes, a criogenia, ou seja, a ciência e a tecnologia de baixas temperaturas, é uma área em desenvolvimento que demanda recursos significativos e envolve processos mecânicos e térmicos complexos.

Onnes realizava um estudo sistemático sobre o comportamento da resistividade elétrica dos metais em baixas temperaturas quando descobriu a supercondutividade. Em razão de suas contribuições à física e tecnologia de baixas temperaturas, ele recebeu o prêmio Nobel de 1913 (ver Quadro 1). Num

\section{FIGURA 1}

Representação esquemática da resistividade elétrica do mercúrio metálico, que apresenta uma transição ao estado supercondutor em $\mathrm{T}_{c}=4,15 \mathrm{~K}$, e da resistividade da prata, que é um metal normal em baixas temperaturas.

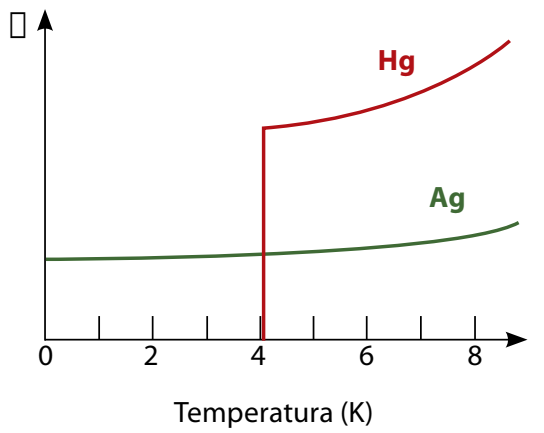

primeiro momento acreditou-se que a supercondutividade seria um fenômeno raro e peculiar ao mercúrio. Porém, pouco tempo após a descoberta, o próprio Onnes mostrou que outros metais se tornam supercondutores quando resfriados a temperaturas suficientemente baixas. Sabemos hoje que cerca de metade dos metais da tabela periódica são supercondutores. Em vários casos, o estado supercondutor é obtido mediante a aplicação de altas pressões. Alguns elementos não me-
1 Um relato histórico sobre a descoberta da supercondutividade, baseado em conversações com o assistente técnico de Kamerlingh Onnes, Gerrit Jan Flim, encontra-se em artigo de Jacobus de Nobel em Physics Today, setembro de 1996. Ver tambémartigo dePeter Kes, nesta edição.

\section{7}

- Karl Alex Müller

- J. Georg Bednorz

Descoberta da supercondutividade de alta temperatura crítica num composto contendo lantânio, bário, cobre e oxigênio

\begin{tabular}{|c|c|c|}
\hline $\begin{array}{c}\text { •Vitaly } \\
\text { Ginzburg } \\
\text { Desenvolvimento } \\
\text { da teoria } \\
\text { fenomenológica da } \\
\text { supercondutividade, } \\
\text { hoje conhecida } \\
\text { como teoria de } \\
\text { Ginzburg-Landau }\end{array}$ & $\begin{array}{c}\text { - Alexei A. } \\
\text { Abrikosov } \\
\text { Teoria dos } \\
\text { vórtices em } \\
\text { supercondutores } \\
\text { de tipo II }\end{array}$ & $\begin{array}{l}\text { Anthony Legget } \\
\text { também foi } \\
\text { contemplado pelo } \\
\text { desenvolvimento } \\
\text { da teoria } \\
\text { da superfluidez } \\
\text { no }{ }^{3} \mathrm{He}, \text { que } \\
\text { é um fenômeno } \\
\text { análogo à } \\
\text { supercondutividade }\end{array}$ \\
\hline
\end{tabular}


tálicos também se tornam supercondutores sob altas pressões. Além dos metais simples, um número muito grande e crescente de ligas e compostos intermetálicos apresenta supercondutividade. Muitos sistemas complexos, com propriedades físicas e químicas variadas, também se tornam supercondutores. Dentre esses estão os cupratos de alta temperatura crítica, os quais serão objeto de discussão mais adiante.

\section{PRINCIPAIS PROPRIEDADES DOS SUPERCONDUTORES}

O estado supercondutor é fortemente afetado pela aplicação de um campo magnético. A presença de um campo magnético tende a produzir uma diminuição na temperatura crítica. Se a magnitude do campo magnético exceder um dado valor característico, denominado campo magnético crítico, $B_{\mathrm{c}}$, o estado supercondutor é suprimido e o material passa a apresentar um comportamento resistivo normal, como aquele ilustrado pela prata na Figura 1. Além disso, se a corrente elétrica que percorre o supercondutor exceder uma dada intensidade denominada de corrente crítica, a supercondutividade também será suprimida

A ausência de resistividade elétrica é a manifestação mais conhecida e espetacular

\section{FIGURA 2}

Contribuição dos elétrons de condução ao calor específico de um metal supercondutor, comparada com o calor específico eletrônico do mesmo metal no estado normal. A anomalia observada na temperatura crítica indica a ocorrência de uma transição de fase envolvendo o sistema de elétrons de condução do material.

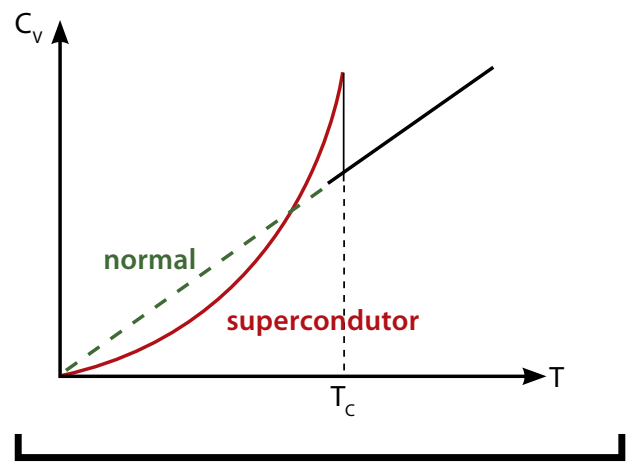

do estado supercondutor. Trata-se, também, da propriedade mais importante do ponto de vista prático. Nota-se, contudo, que a resistividade será rigorosamente nula somente se a corrente aplicada ao material for contínua. Se o supercondutor for percorrido por uma corrente alternada, dissipação poderá ser observada se a frequência da corrente for suficientemente alta.

\section{QUADRO 1}

\section{CALOR ESPECÍFICO DE UM METAL NORMAL}

$\mathrm{Q}$ uando uma dada quantidade de energia (calor) é transferida a um sistema termodinâmico (um sólido, por exemplo), este responde através de uma elevação em sua temperatura. Supondo que o sistema está termicamente isolado de sua vizinhança, a razão entre o calor cedido por unidade de massa (ou por unidade de volume) da substância e a correspondente variação da temperatura é denominada de calor específico, o qual é uma propriedade da substância. O calor cedido ao material produz um aumento em sua energia interna. Num sólido metálico, as principais contribuições à energia interna estão relacionadas às oscilações dos átomos em torno de suas posições na rede cristalina e ao movimento dos elétrons de condução.Correspondentemente, existem duas contribuições aditivas ao calor específico. Em baixas temperaturas, ambas as contribuições dependem da temperatura segundo leis de potência simples e podem ser facilmente identificadas e separadas. No estado metálico normal, a contribuição dos elétrons de condução é linearmente proporcional aT, conforme mostraa Figura 2, enquanto a contribuição devida às oscilações da estrutura cristalina é proporcional $\mathrm{T}^{3}$. 
Efeito Meissner-Ochsenfeld. (a) Em temperaturas superiores a $\mathrm{T}_{\mathrm{c}^{\prime}}$ um campo magnético aplicado externamente permeia um material supercondutor. (b) Em temperaturas inferiores a $T_{c}$ a indução magnética é expulsa do interior da amostra supercondutora. (c) Representação da indução magnética no interior do material $\left(B_{i}\right)$ em função do campo externo (B). Em $B=B_{c^{\prime}}$ a supercondutividade é destruída.

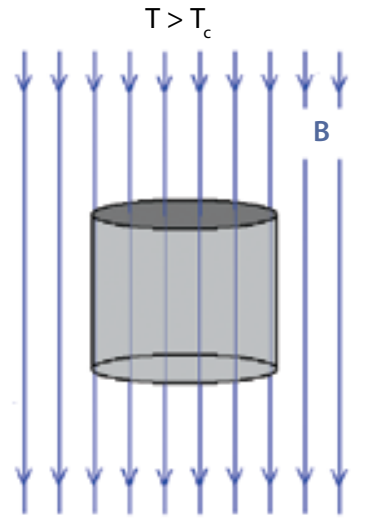

(a)

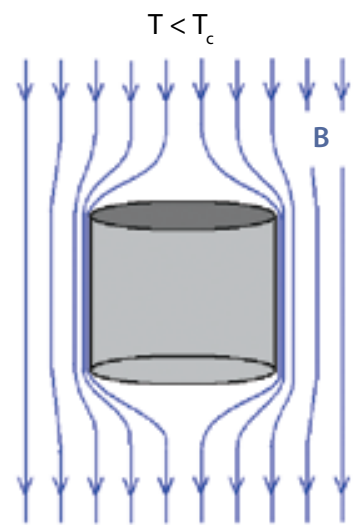

(b)

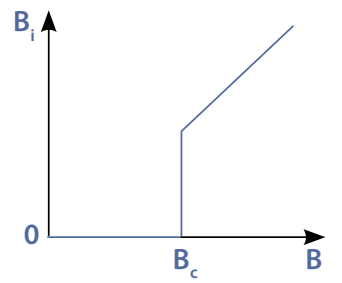

(c)
Muitas outras propriedades físicas revelam a presença de supercondutividade. Dentre elas está o calor específico (ver Quadro 1). A contribuição dos elétrons de condução ao calor específico de um supercondutor apresenta um comportamento peculiar, conforme está mostrado esquematicamente na Figura 2. Na temperatura crítica, o calor específico eletrônico sofre uma variação abrupta e, em baixas temperaturas, tende a zero segundo uma lei exponencial. $\mathrm{O}$ comportamento observado nas vizinhanças de $T_{\mathrm{c}}$ é conhecido como anomalia do tipo lambda, em razão da semelhança com a letra grega de mesmo nome. Num metal normal, o calor específico eletrônico é uma função linear da temperatura e pode ser observado quando o sistema supercondutor é submetido a um campo magnético de magnitude superior ao valor crítico.

O calor específico é uma propriedade de equilíbrio termodinâmico. Assim, a anomalia lambda observada na temperatura crítica é originada pela ocorrência de um fenômeno de transição de fase envolvendo o sistema eletrônico do material supercondutor. Acima de $T_{\mathrm{c}}$, observa-se um comportamento eletrônico característico de um metal normal, ao passo que, em temperaturas inferiores a $T_{\mathrm{c}}$, os elétrons de condução se organizam num outro estado termodinâmico: o estado supercondutor. $\mathrm{O}$ fenômeno que ocorre em $T_{\mathrm{c}}$, portanto, é uma transição de fase termodinâmica causada pela variação da temperatura. Transições de fase são frequentes na natureza. Um exemplo corriqueiro é a mudança da água em gelo, que ocorre em $0^{\circ} \mathrm{C}$.

Outra propriedade muito importante do estado supercondutor é o efeito Meissner-Ochsenfeld, assim denominado em homenagem aos seus descobridores, os cientistas alemães Walther Meissner e Robert Ochsenfeld ${ }^{2}$. Na maioria dos textos, no entanto, a propriedade é denominada simplesmente como efeito Meissner, o qual está ilustrado na Figura 3. O painel (a) dessa figura mostra que um campo magnético aplicado permeia totalmente um material supercondutor quando este se encontra no estado normal. Porém, se a temperatura for diminuída, observa-se a expulsão abrupta das linhas de indução do campo do interior do material durante a tran-
20 efeito Meissner-Ochsenfeld foi observado pela primeira vez em 1933. Um relato detalhado dos antecedentes e dos fatos associados a essa descobertapode ser encontrado em $\mathrm{F}$. Dahl (1992). 
3 Uma comparação detalhada entre os comportamentos magnéticos de um supercondutor e de um metal normal, ao qual se aplicam somente as leis do eletromagnetismo clássico, pode ser encontrada em Supercondutividade (Ostermann\& Pureur, 2005). sição ao estado supercondutor, em $T=T$, tal como mostra o painel (b).

O efeito Meissner é uma manifestação da natureza quântica do estado supercondutor e não tem análogo na física clássica. O campo magnético é expulso de dentro do supercondutor mesmo que se trate de um campo estático. Esse fato não pode ser explicado pela lei da indução do eletromagnetismo clássico ${ }^{3}$.

O comportamento magnético dos supercondutores permite a classificação desses sistemas em dois tipos. Nos supercondutores do tipo I, o efeito Meissner é completo, ou seja, o fluxo magnético é totalmente expulso do interior da amostra, conforme mostra a Figura 3. Essa situação permanece até que o valor do campo aplicado atinja o valor crítico $B_{c}$. Então, como se observa no gráfico do painel (c), a supercondutividade é suprimida bruscamente, o efeito Meissner cessa, e o campo volta a penetrar livremente no material. Os elementos metálicos, em estado muito puro, são supercondutores do tipo I.

\section{FIGURA 4}

(a) Efeito Meissner-Ochsenfeld parcial num supercondutor do tipo II. (b) Campo interno em função do campo aplicado: o campo magnético é totalmente expulso somente até o campo crítico inferior $B_{c 1}$. No campo crítico superior, $\mathrm{B}_{\mathrm{c} 2^{\prime}}$ a supercondutividade é destruída. No estado entre $B_{c 1}$ e $B_{c 2^{\prime}}$ o campo magnético penetra na amostra na forma de vórtices quantizados.

$$
\mathrm{B}_{\mathrm{c} 1}<\mathrm{B}<\mathrm{B}_{\mathrm{c} 2}
$$

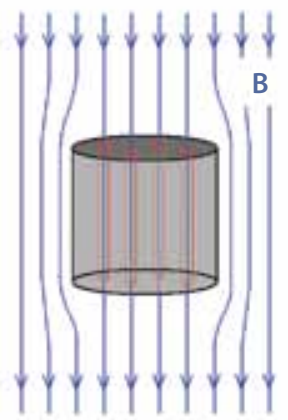

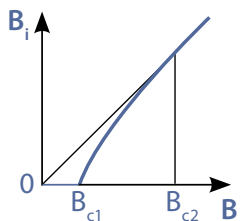

(b) (a)

\section{FIGURA 5}

Pequena amostra supercondutora levitando sobre polo magnético.

A força magnética repulsiva ( $F m$ ) gerada pelo efeito

Meissner equilibra o peso (P).

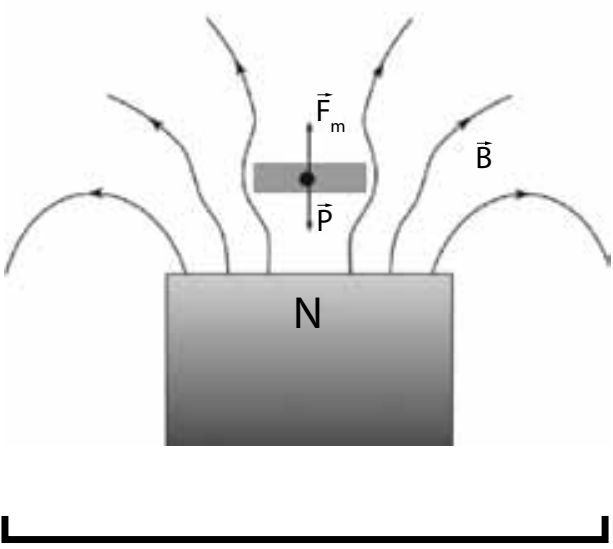

A grande maioria dos supercondutores é do tipo II. Nesse caso, há dois valores críticos para o campo magnético. A expulsão total do fluxo ocorre somente se a magnitude do campo aplicado for menor que o campo crítico inferior, característico do material, e denotado como $B_{c 1}$ no gráfico do painel (b) da Figura 4. O campo magnético penetra parcialmente na amostra, sem destruir o estado supercondutor, se seu valor for superior a $B_{\mathrm{cl}}$ e inferior ao campo crítico superior $B_{c 2}$. A Figura 4 (a) ilustra esquematicamente a expulsão parcial do fluxo magnético e a penetração do campo na região de coexistência de campo e supercondutividade. Se o campo magnético externo tiver intensidade igual ou superior a $B_{c 2}$, o estado supercondutor é destruído. $\mathrm{O}$ campo crítico superior tem valores muito elevados em vários materiais supercondutores de tipo II. Em regra, esses são os sistemas com maior interesse para uso tecnológico.

O efeito Meissner é responsável pelo fascinante e popular fenômeno da levitação de um pequeno ímã sobre uma peça de material supercondutor, ou, de modo equivalente, de uma amostra supercondutora 
sobre a superfície de um polo de material magnético, conforme ilustração da Figura 5. A expulsão do campo do interior do supercondutor é acompanhada pela geração de "supercorrentes" superficiais ao material, que produzem uma magnetização cujo sentido é oposto ao do campo magnético externo. Assim, uma interação repulsiva é estabelecida entre o supercondutor e o material magnético. Essa interação é sempre repulsiva, independentemente do sinal do polo magnético próximo ao supercondutor, e pode ser suficientemente forte para compensar a força gravitacional e produzir levitação, como esquematizado na figura.

\section{VÓRTICES EM SUPERCONDUTORES DE TIPO II}

A penetração do campo magnético nos supercondutores de tipo II é um fenômeno admirável. O campo se subdivide em estru-

\section{FIGURA 6}

(a) Vórtices em supercondutores de tipo II. Cada vórtice é atravessado por um quantum de fluxo magnético

$\left(\varphi=2,0710^{-15} \mathrm{~T} \mathrm{~m}^{2}\right)$. Em torno dos caroços filamentares circulam correntes de blindagem ao campo magnético.

(b) Os vórtices formam uma rede de simetria triangular.
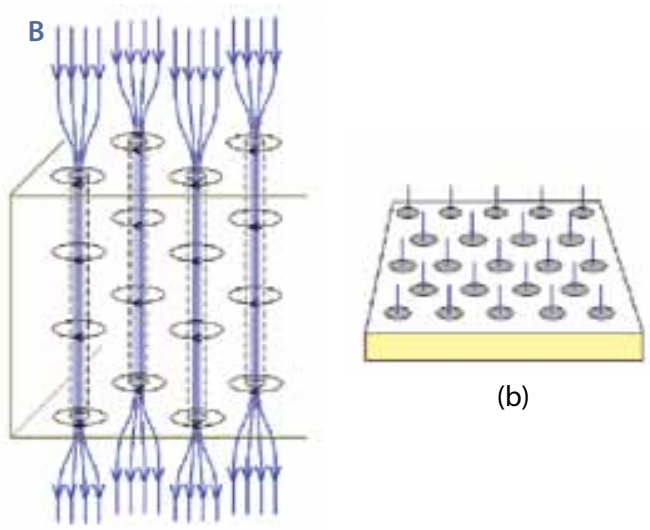

(b) turas filamentares, denominadas vórtices, que são regularmente distribuídas e atravessam a amostra de um lado a outro conforme está esquematizado na Figura 6. Na região central do vórtice, cujo diâmetro é da ordem de décimos de micrômetros, a supercondutividade é suprimida. Esse caroço normal é atravessado por um quantum de fluxo magnético (em unidades do sistema internacional, um quantum de fluxo magnético para o estado supercondutor tem o valor $\varphi=2,07$ $10^{-15}$ tesla. $\mathrm{m}^{2}$ ). Em torno do caroço circulam correntes supercondutoras, formando uma estrutura semelhante a um torvelinho que observamos quando um fluido escoa através de um orifício. As correntes supercondutoras circulares impedem a penetração do campo magnético em regiões da amostra que estejam afastadas do vórtice (ver painel (a) da Figura 6). Em razão de repulsão mútua, os vórtices se arranjam numa estrutura periódica, com simetria triangular, conforme esquematizado na Figura 6 (b). Quanto mais intenso for o campo aplicado, maior será o número de vórtices, pois cada um deles é atravessado por um único quantum de fluxo magnético. A supercondutividade é globalmente suprimida quando a densidade de vórtices é tal que seus caroços normais entram em contato uns com os outros. A magnitude do campo magnético aplicada será, então, igual à do campo crítico superior.

Os vórtices são genuínas estruturas quânticas que se manifestam em escala macroscópica. A formação do estado de vórtices em supercondutores de tipo II foi prevista teoricamente por Andrei Abrikosov, em 1956. Em razão dessa previsão, Abrikosov foi contemplado com o prêmio Nobel de 2003.

\section{O DESAFIO DA TEORIA MICROSCÓPICA}

A teoria básica, que fundamenta a descrição do comportamento dos elétrons de condução num sistema metálico normal, considera que essas partículas se movimentam como se fossem objetos independentes uns dos outros. Assim, por exemplo, ao se 
4 Uma revisão crítica e histórica dos fatos que precederam a teoria BCS e as etapas de seu desenvolvimento foi recentemente publicada no texto $B C S$ : 50 Years, editado por Leon Cooper e Dmitri Feldman, World Scientific, Singapura, 2011. propagar no interior do metal sob ação de um campo elétrico, os elétrons de condução cedem energia ao se chocar (interagir, na linguagem da teoria quântica) com imperfeições estáticas e dinâmicas da estrutura cristalina. Essas interações se manifestam, macroscopicamente, no comportamento resistivo, que é comum a todos os metais percorridos por correntes elétricas. Em razão desse entendimento, foi extremamente difícil e demorado conceber um quadro teórico de primeiros princípios que pudesse descrever o desaparecimento completo da resistividade elétrica de certos sistemas metálicos em temperaturas inferiores a um valor crítico. Os 46 anos transcorridos entre a descoberta da supercondutividade e a descrição teórica do fenômeno são justificados pela superação de muitas dificuldades conceituais e desafios metodológicos. Os autores da teoria microscópica da supercondutividade, John Bardeen, Leon Cooper e Robert Schrieffer, receberam

\section{FIGURA 7}

As oscilações coerentes da rede geram regiões onde ocorre momentaneamente um acúmulo de íons positivamente carregados. Esses desequilíbrios de carga positiva podem causar uma interação efetivamente atrativa entre elétrons e assim estabilizar pares de Cooper.

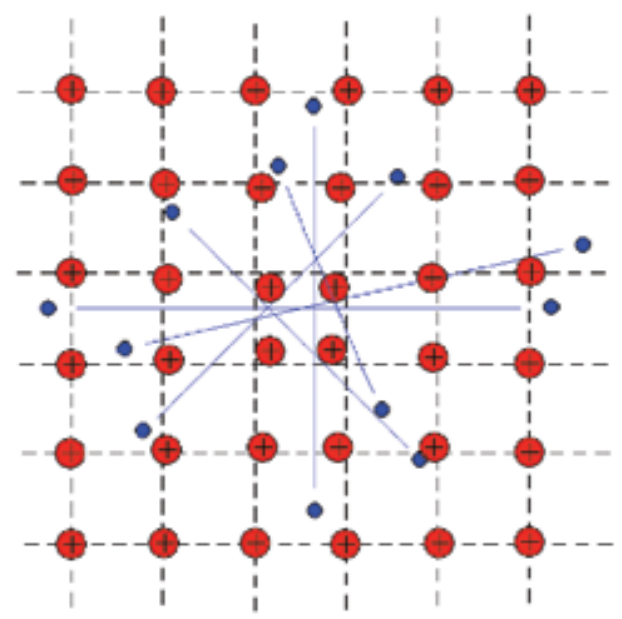$$
\text { L }
$$

o prêmio Nobel de 1972 (Bardeen, Cooper \& Schriffer, 1957). Em homenagem a eles, essa teoria é hoje conhecida como teoria BCS, acrônimo de seus sobrenomes ${ }^{4}$.

A ideia fundamental da teoria BCS consiste na suposição de que os elétrons de condução mais energéticos se associam em pares com velocidades opostas e spins também opostos (para o presente propósito, o spin de um elétron pode ser entendido como a propriedade responsável pelo momento magnético intrínseco da partícula; assim, dizer que os spins são opostos significa que os momentos magnéticos intrínsecos dos elétrons componentes do par apontam em sentidos opostos). A associação dos elétrons em pares, chamados de pares de Cooper, se dá em razão de uma interação efetivamente atrativa entre os componentes do par. Essa atração, que deve superar a repulsão natural entre partículas de mesma carga elétrica, é mediada pelas vibrações da rede cristalina. A Figura 7 é uma representação esquemática desse processo indireto de atração entre elétrons que leva à formação de pares fracamente ligados, os quais ocupam extensas regiões no interior do sólido. Assim, o volume espacial ocupado por um par de Cooper contém os centros de massa de um número muito grande de outros pares de Cooper (em certos elementos supercondutores, esse número pode alcançar centenas de milhares, ou milhões). Isso significa que os pares de Cooper, embora formados por elétrons de condução, não podem ser considerados como entidades isoladas. Em outros termos, os pares de Cooper formam um estado de elétrons interdependentes cujos movimentos são complexamente imbricados uns aos outros. O condensado resultante se comporta como um coletivo quântico que se estende por escalas de tamanho macroscópico. Assim, quando posto em movimento pela ação de um campo elétrico, o condensado supercondutor avança sem ser detido por eventos que, embora capazes de degradar a propagação de elétrons independentes, não possuem energia suficiente para afetar o condensado globalmente. Dessa forma, pode-se entender 
por que, num supercondutor, uma corrente elétrica se propaga sem encontrar resistência.

A teoria BCS também descreve adequadamente o campo magnético crítico, a anomalia do calor específico, o efeito Meissner-Ochsenfeld, e muitas outras propriedades do estado supercondutor.

\section{O DESAFIO DAS ALTAS TEMPERATURAS CRÍTICAS}

Durante mais de setenta anos, o fenômeno da supercondutividade ficou restrito ao domínio das temperaturas muito baixas. A teoria BCS, em sua formulação original, favorecia a interpretação de que a supercondutividade seria uma propriedade observável apenas em temperaturas próximas ao zero absoluto. Esse era o cenário em 1986, quando K. Alex Müller e J. Georg Bednorz reportaram a observação de supercondutividade em temperaturas superiores a $30 \mathrm{~K}$ num composto óxido contendo lantânio, bário, cobre e oxigênio. $\mathrm{O}$ sistema original, de fórmula química $\mathrm{La}_{2} \mathrm{CuO}_{4}$, é um isolante elétrico, como geralmente são os compostos óxidos no estado sólido. Porém, a substituição parcial de lantânio por bário torna o sistema um condutor elétrico e, mais do que isso, um supercondutor com temperatura crítica $T_{c}=36$ K. O modesto artigo de Müller e Bednorz (1986, p. 189), à época pesquisadores de um laboratório de uma grande empresa privada (IBM), em Zurique, Suíça, inicialmente não causou impacto na comunidade científica. No entanto, quando os resultados foram reproduzidos e melhorados por uma reputada equipe de pesquisadores japoneses, $\mathrm{o}$ interesse científico pelo tema da supercondutividade renasceu com grande vigor. Começava, então, uma das mais trepidantes eras, em todos os tempos, da pesquisa científica e tecnológica em materiais sólidos. Uma extraordinária mudança de paradigma no que se refere ao valor das temperaturas críticas se consolidou em alguns poucos anos (ver Quadro 2). O trabalho pioneiro de Müller e Bednorz foi re- conhecido, com surpreendente rapidez, através da concessão do prêmio Nobel de 1987.

No início de 1987, um grupo de pesquisadores das universidades de Houston (Texas) e do Alabama, liderados por C. W. (Paul) Chu, reportou a observação de supercondutividade num sistema contendo ítrio, bário, cobre e oxigênio, no qual a temperatura crítica alcançava estonteantes (para a época) $91 \mathrm{~K}$ (Wu et al., 1987, p. 908). A ideia que produziu esse resultado foi tão somente a substituição do lantânio por ítrio na composição do sistema investigado por Müller e Bednorz. O ítrio ocupa a posição logo acima do lantânio, na mesma coluna da tabela periódica. Os dois átomos possuem tamanhos distintos, porém suas propriedades químicas são semelhantes. Os resultados de Chu e seus colaboradores produziram um impacto extraordinário na comunidade científica, que logo alcançou o grande público pela via da mídia ordinária. $\mathrm{O}$ material descoberto por essa equipe, cuja fórmula química é $\mathrm{YBa}_{2} \mathrm{Cu}_{3} \mathrm{O}_{7}$, popularmente conhecido como YBCO, ou ybacuo, tornou-se um dos sistemas mais estudados de todos os tempos. A razão da tremenda excitação causada por sua descoberta estava relacionada ao fato de que a temperatura crítica do YBCO excede a temperatura de ebulição do nitrogênio líquido (77 K). Isso significava que, pela primeira vez, dispunha-se de um material cujo estado supercondutor podia ser alcançado com métodos incomparavelmente mais simples e menos dispendiosos que os necessários para resfriar os materiais metálicos convencionais, hoje ditos supercondutores clássicos. Portanto, a descoberta do YBCO potencialmente abria a possibilidade de ampla aplicação prática do fenômeno da supercondutividade. De fato, à época, era comum se ouvir falar de revolução tecnológica produzida pela supercondutividade.

Num curto intervalo de tempo, diversos outros materiais supercondutores de alta temperatura crítica foram descobertos. O recorde alcançou $T_{\mathrm{c}}=133 \mathrm{~K}$, num composto de estrutura cristalina complexa contendo mercúrio, bário, cálcio, cobre e oxigênio. O traço comum desses óxidos, hoje denominados de 
cupratos supercondutores, é a presença de planos atômicos formados por cobre e oxigênio, na proporção $\mathrm{Cu}-\mathrm{O}_{2}$. Essas camadas se repetem periodicamente e constituem o "coração eletrônico" do sistema. Na Figura 8 está uma representação esquemática da estrutura cristalina do YBCO. Nesse composto, os planos de $\mathrm{Cu}-\mathrm{O}_{2}$ ocorrem em pares que são separados por planos atômicos de ítrio. As estruturas de duplos planos de $\mathrm{Cu}-\mathrm{O}_{2}$ são intercaladas por outras camadas atômicas, que atuam como reservatórios de cargas, os quais liberam partículas portadoras de carga para os planos condutores.

Embora um quarto de século tenha se passado desde a descoberta de Müller e Bednorz, o entendimento das propriedades eletrônicas dos cupratos supercondutores ainda represen-

\section{FIGURA 8}

Estrutura cristalina (esquemática) do YBCO. A estrutura de duplos planos atômicos de $\mathrm{Cu}-\mathrm{O}_{2}$ e as camadas que atuam como reservatório de carga estão indicadas.

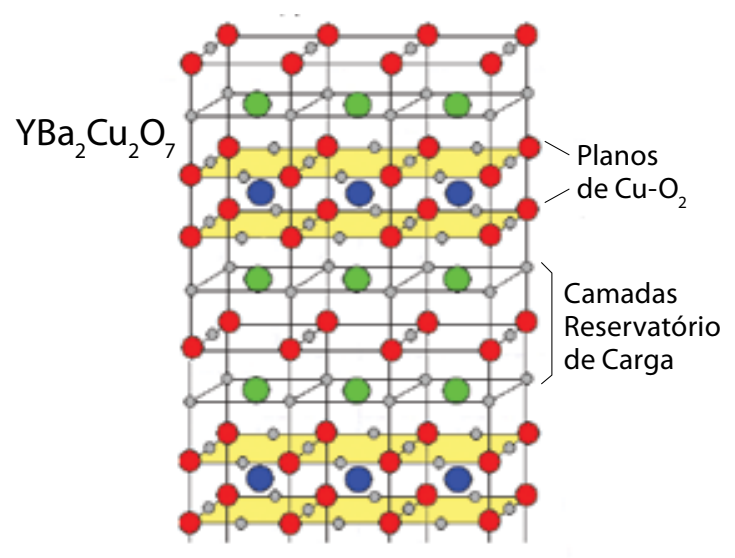

- Oxigênio

- Cobre

Bário

Ítrio ta um grande desafio científico. Não há consenso sequer sobre a natureza do mecanismo que leva à formação dos pares de Cooper nesses sistemas. Diversos argumentos sugerem que a interação indireta, mediada pelas vibrações da rede cristalina e responsável pelo pareamento nos supercondutores clássicos, por si só, seria incapaz de assegurar a estabilidade de um par de Cooper em temperaturas da ordem de $100 \mathrm{~K}$. Assim, diversos cenários envolvendo excitações de natureza puramente eletrônica têm sido propostos. Contudo, até o momento, nenhum deles logrou reproduzir todos os aspectos experimentais conhecidos.

Além das dificuldades relativas à descrição do estado supercondutor, a compreensão das propriedades desses sistemas na fase normal também se constitui num dos mais relevantes problemas da física do estado sólido contemporânea. Os cupratos, segundo o entendimento corrente, não são metais no sentido usual. Esses sistemas derivam de fases isolantes e magnéticas. Por meio de um processo denominado de dopagem, produzido pela substituição atômica, como no caso do La-Ba-Cu-O, ou pela variação no conteúdo de oxigênio, como no caso do YBCO, uma fração dos elétrons de valência se desvincula dos átomos de origem. Tais elétrons ficam aptos a transportar corrente. Porém, entre eles subsistem fortes correlações mútuas. Assim, não é adequado descrever o movimento dos elétrons de condução nos cupratos supercondutores segundo a concepção válida para os metais clássicos, em que tais partículas podem ser descritas como elétrons independentes e quase livres.

Contrastando com a temática científica polêmica e desafiadora, os cupratos têm decepcionado do ponto de vista tecnológico. Em razão da instabilidade química e de propriedades mecânicas desfavoráveis, tem sido difícil conformar esses materiais em dispositivos práticos. Um exemplo dessas dificuldades é a produção de fios. O desafio consiste, em termos simples, na transformação de uma cerâmica dura e quebradiça em fios flexíveis e contínuos, com propriedades homogêneas em extensões da ordem de alguns quilômetros. 


\section{OS NOVOS DESAFIOS CIENTÍFICOS}

A descoberta dos cupratos supercondutores revolucionou a área de pesquisa em supercondutividade. Uma das lições mais importantes trazidas pelo estudo desses materiais é a descoberta de que a supercondutividade não é necessariamente um fenômeno confinado ao mundo gelado das temperaturas próximas ao zero absoluto. Portanto, não há razões de princípio que desaconselhem a busca por materiais supercondutores que tenham temperaturas críticas ainda mais elevadas e, quiçá, possuam propriedades químicas e mecânicas mais favoráveis às aplicações tecnológicas.

A linha de pesquisa em novos materiais supercondutores levou à descoberta de alguns sistemas notáveis em tempos recentes. Esse é, por exemplo, o caso do diboreto de magnésio $\left(\mathrm{MgB}_{2}\right)$. Nesse sistema simples ver estrutura cristalina na Figura 9(a) -, a ocorrência de supercondutividade com temperatura crítica $T_{\mathrm{c}}=39 \mathrm{~K}$ foi reportada em 2001 (Nagamatsu et al., 2001, p. 63). O valor da temperatura crítica desse composto intermetálico é significativamente maior do que os valores típicos para os supercondutores clássicos (ver Quadro 2). Esse fato indica que a complexidade estrutural dos cupratos não é, necessariamente, um requisito indispensável à obtenção de temperaturas críticas elevadas.

Recentemente, uma descoberta surpreendente produziu grande impacto na comunidade de especialistas em supercondutividade. Em 2008, um grupo de pesquisadores do Instituto de Tecnologia de Tóquio, liderados por H. Hosono, anunciou a observação de supercondutividade com $T_{c}=26 \mathrm{~K}$ num sistema contendo átomos de lantânio, ferro, arsênio, oxigênio e flúor ${ }^{5}$. Logo a seguir, modificações na composição química do composto original levaram à obtenção de famílias de compostos supercondutores, genericamente apelidados de pnictídeos, ou ferro-pnictídeos, nos quais a característica estrutural comum é a presença de planos atômicos formados por átomos de ferro e arsênio, ou ferro e selênio. Temperaturas críticas superiores a $50 \mathrm{~K}$ foram reportadas num material contendo samário, ferro, arsênio e oxigênio dopado com uma pequena quantidade de flúor. Uma variante de grande interesse entre os ferro-pnictídeos é o sistema de fórmula química $\mathrm{Ba}_{1-\mathrm{x}} \mathrm{K}_{\mathrm{x}} \mathrm{Fe}_{2} \mathrm{As}_{2}$ - ver estrutura cristalina na Figura 9(b) -, no qual uma pequena quantidade de bário é substituída por átomos de potássio. Embora a temperatura crítica, nesse caso, alcance valores pouco superiores a 30 $\mathrm{K}$, amostras desse composto podem ser crescidas na forma de monocristais, o que favorece o estudo detalhado de suas propriedades físicas. $\mathrm{O}$ fato surpreendente relacionado aos pnictídeos é a grande quantidade de átomos de ferro presente nos compostos. Até então, o ferro era considerado como o inimigo nú-

QUADRO 2

\section{EVOLUÇÃO CRONOLÓGICA DA TEMPERATURA CRÍTICA DOS MATERIAIS SUPERCONDUTORES}

O período clássico, dominado pelos supercondutores metálicos, estende-se desde a descoberta da supercondutividade até 1986 , ano da descoberta do primeiro cuprato supercondutor de alta temperatura crítica. As temperaturas do hélio

líquido e do nitrogênio líquido, marcos tecnológicos, estão assinaladas.

Dois representantes dos supercondutores pós-cupratos estão também assinalados.

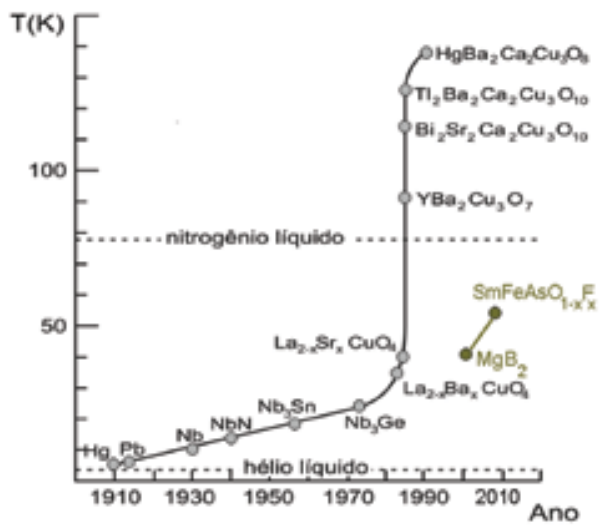

5 Uma revisão sobre as propriedades dosnovíssimossupercondutoresferro-pnictídeos é encontrada em Hosono, 2009. 
mero um da supercondutividade. De fato, o ferro é o elemento mais representativo dos materiais magnéticos e seu nome dá origem à denominação do ordenamento ferromagnético, característico dos ímãs poderosos. Como vimos na parte inicial deste texto, a supercondutividade não coexiste facilmente com o magnetismo. Esses fenômenos são, em geral, excludentes. Assim, a entrada em cena dos ferro-pnictídeos representa um novo e grande desafio de natureza fundamental, que se relaciona à elucidação do mecanismo que leva à cooperação entre magnetismo e supercondutividade em certas circunstâncias. Trata-se, no caso dos pnictídeos, de entender por que a presença do ferro não é nociva à supercondutividade. Pelo contrário, são as camadas atômicas formadas por ferro e arsênio (ou selênio), evidentes na estrutura cristalina mostrada na Figura 9(b), que se constituem no subsistema controlador das propriedades eletrônicas desses compostos.

Os pnictídeos supercondutores e, em menor medida, o $\mathrm{MgB}_{2}$ também demonstram que a presença de oxigênio, ou de planos atômicos de $\mathrm{Cu}-\mathrm{O}_{2}$, não é indispensável à estabilização de supercondutividade em temperaturas elevadas. Essa é mais uma razão para se manter o otimismo quanto à descoberta de novos supercondutores, com variadas propriedades, cuja caracterização e interpretação representem desafios que impulsionem o avanço do conhecimento científico e tecnológico.

\section{O DESAFIO TECNOLÓGICO}

O fenômeno da supercondutividade proporcionou a concepção e o desenvolvimento de diversas aplicações tecnológicas. Tais dispositivos, em grande maioria, são confeccionados com supercondutores metálicos clássicos. Portanto, para torná-los operacionais é necessário resfriá-los até temperaturas próximas ao zero absoluto. $\mathrm{O}$ custo e as dificuldades técnicas inerentes à obtenção e manutenção por longos intervalos de tempo de temperaturas muito baixas tornam inviável economicamente o uso generalizado da tecnologia baseada na supercondutividade. Apenas em situações específicas, as complicações relacionadas à criogenia são compensadas pela performance e benefícios que se

\section{FIGURA 9}

(a) Estrutura cristalina hexagonal do supercondutor $\mathrm{MgB}_{2}$. (b) Estrutura cristalina em camadas do picnitídeo supercondutor $\mathrm{Ba}_{1-x} \mathrm{~K}_{x} \mathrm{Fe}_{2} \mathrm{As}_{2}$.

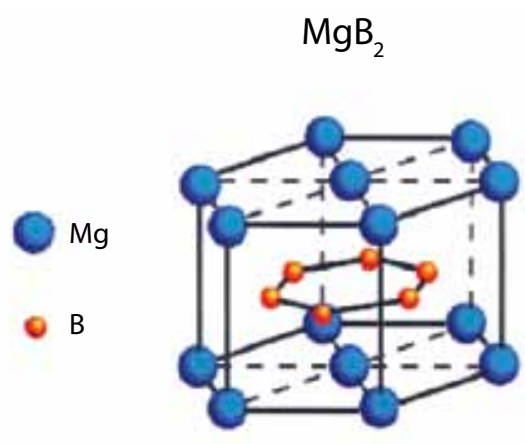

(a)

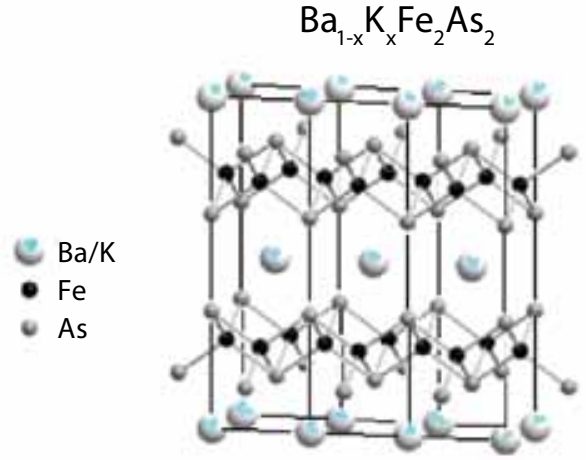

(b)

1 
FIGURA 10

Princípio de operação de um trem Maglev

Eletroímãs convencionais para guia, estabilidade e propulsão (no trem)

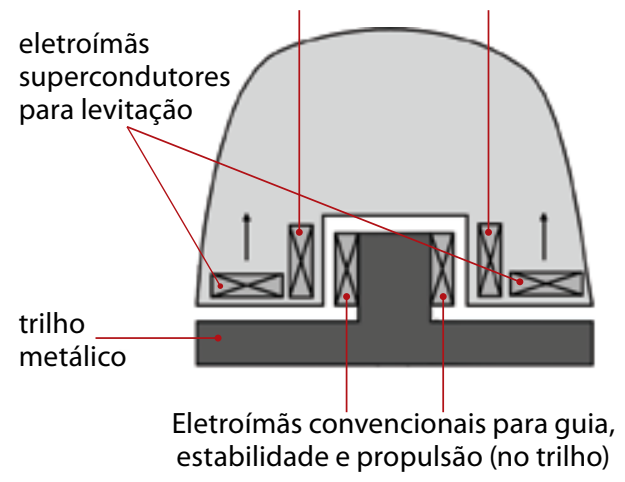

pode obter de dispositivos supercondutores.

Estima-se que, num prazo aproximado de quinze anos, a área de energia concentrará a maior parte das aplicações da supercondutividade. A transmissão de energia sem perdas por aquecimento resistivo dos fios condutores é uma possibilidade há muito considerada. $\mathrm{O}$ armazenamento de energia em campo magnético intenso é outra possibilidade interessante. Para tanto, tem sido proposta a utilização de grandes toroides, enrolados com fios supercondutores, com os quais é possível produzir campos magnéticos intensos que funcionam como reservatórios de energia limpa e facilmente recuperável. Tais sistemas, denominados SMES (do inglês superconducting storage energy system), poderiam funcionar armazenando a energia em excesso produzida por centrais convencionais em períodos de baixo consumo (à noite, por exemplo) e liberá-la durante os picos de maior demanda. O conceito de SMES foi originalmente concebido para funcionar como um tipo de no-break, que poderia ter escala de tamanho para atender uma indústria, por exemplo, ou até uma cidade inteira.

A geração de campos magnéticos intensos e controláveis com bobinas confeccio- nadas com fios supercondutores tem muitas outras aplicações, presentes e potenciais. A utilização de tais sistemas em laboratórios de pesquisa data de várias dezenas de anos. Mais recentemente, campos magnéticos intensos, gerados por supercondutores, têm encontrado aplicações na área médica ${ }^{6}$, principalmente em tomografia por ressonância magnética nuclear. Campos magnéticos intensos também são usados em dispositivos de levitação. Assim, aplicações em transporte público de massa, tais como trens Maglev (Figura 10), que flutuam sobre os trilhos e se deslocam em alta velocidade, estão evoluindo do estágio de protótipo para operação em escala real.

A transmissão de energia e a geração de campos magnéticos exigem a conformação dos materiais supercondutores em fios de comprimento quilométrico, de modo que seja garantida a homogeneidade de propriedades ao longo de tais extensões. Os materiais mais utilizados na produção de fios supercondutores são a liga nióbio-titânio e o composto intermetálico $\mathrm{Nb}_{3} \mathrm{Sn}$. Os fios mais eficientes são do tipo multifilamentar, esquematizado na Figura 11. Nesses fios, numerosos filamentos de material supercondutor são embebidos num metal convencional que seja um bom condutor térmico e elétrico. O encapsulamento evita que uma eventual flutuação com perda de supercondutividade num dado ponto de um filamento se propague catastroficamente por todo o fio. Um excesso de corrente momentâneo flui pela matriz metálica que, em operação normal, é "curto-circuitada" pelos filamentos supercondutores. Ademais, o encapsulamento melhora a estabilidade térmica do fio. Com essa tecnologia é possível produzir fios com diâmetro inferior a um milímetro que podem transportar correntes elétricas de 100 amperes, ou mais.

A dificuldade para a produção de fios é o maior entrave ao uso prático dos cupratos supercondutores de alta temperatura crítica. A natureza mecânica dura e quebradiça e as propriedades extremamente anisotrópicas desses sistemas representam obstáculos de difícil superação. Duas estratégias têm sido
6 Verartigo de Oswaldo Baffa e Draulio Barros de Araujo, nesta edição. 
usadas, cujos resultados ainda não são satisfatórios. A mais simples delas consiste na moagem de amostras policristalinas, no preenchimento de tubos metálicos com o pó de material supercondutor, na laminação do tubo transformando-o em fitas e no posterior tratamento térmico das fitas de modo a produzir a sinterização dos grãos em seu interior - ver Figura 12(a). Essa estratégia (denominada PIT, do inglês powder in tube) tem vários inconvenientes. Dentre esses está a corrente crítica medíocre que resulta da sinterização de grãos aleatoriamente orientados. Outro ponto desfavorável é a necessidade da utilização do valioso metal prata na confecção dos tubos em razão da reatividade dos cupratos com outros metais menos dispendiosos. A outra estratégia é mais recente e tem produzido resultados promissores. A ideia consiste no crescimento de filmes finos de cuprato supercondutor (YBCO é o material mais favorável) sobre uma fita metálica especialmente preparada, tal como esquematizado na Figura 12(b). O desenvolvimento de técnicas químicas para deposição de filmes, em princípio, permite que o processo seja realizado em contínuo. Assim, fitas quilométricas podem ser confeccionadas. Para que correntes críticas apreciáveis sejam obtidas, diversas dessas estruturas poderiam ser sobrepostas solidariamente, formando uma fita espessa.

Os supercondutores também podem ser conformados em pequenos dispositivos para aplicação em eletrônica. A estrutura mais interessante é a chamada junção Josephson, esquematizada na Figura 13. Nesse sistema, dois eletrodos supercondutores são separados um do outro por uma finíssima camada de material não supercondutor, que pode ser um isolante elétrico, ou um metal normal. Em tal sistema, estudado por Brian Josephson (ver box sobre prêmio Nobel), pares de Cooper atravessam de um banco supercondutor para o outro através do isolante via processo de tunelamento quântico. Um anel de material supercondutor contendo uma ou duas junções Josephson constitui um dispositivo denominado squid (superconducting quantum interference device), que é extremamente sensível a sinais magnéticos de fraquíssima intensidade. Assim, equipa-

FIGURA 11

Fio multifilamentar de material supercondutor metálico

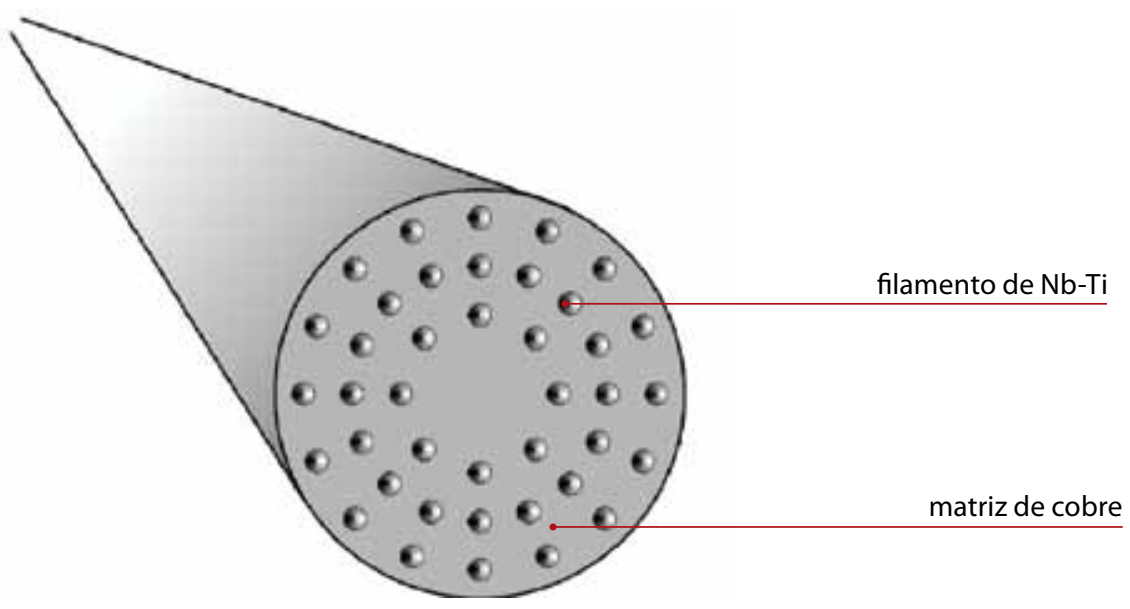

1 


\section{FIGURA 12}

Propostas para confecção de fios dos supercondutores de alta temperatura crítica.

(a) Tecnologia PIT (powder in tube), com posterior conformação em fita. (b) Filme fino supercondutor crescido sobre fita metálica.

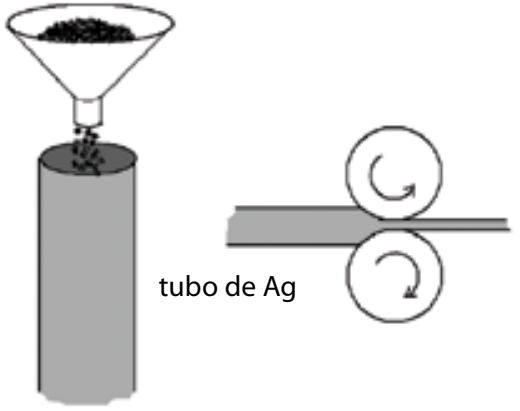

(a)

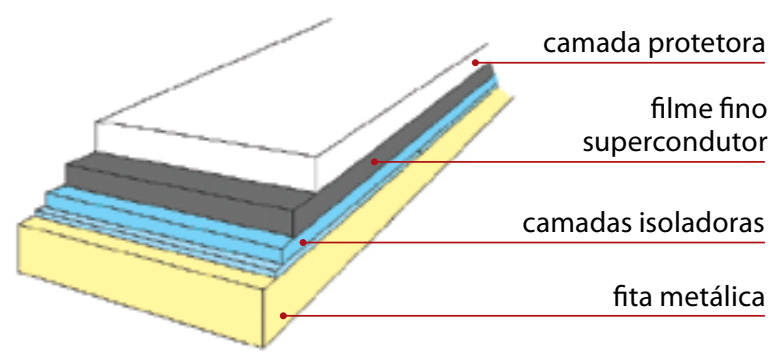

(b)

L

mentos baseados em squids são amplamente empregados na pesquisa de materiais magnéticos em laboratório, na prospecção geológica, na indústria petrolífera e até na área médica, onde atuam como sensores em equipamentos de magnetoencefalografia ${ }^{7}$. Outra aplicação importante das junções Josephson é na geração e detecção sintonizável de radiação eletromagnética na faixa de frequências de centenas de gigahertz. Também digna de atenção é a possibilidade de emprego de redes de junções Josephson como elementos lógicos em computadores quânticos, que teoricamente têm capacidade de processamento muito superior à dos computadores de lógica binária tradicional. Demonstrações da realização e operação de qubits - o elemento básico da arquitetura de computadores quânticos - com base em junções Josephson têm sido reportadas (Shnirman, Schön \& Hernon, 1997, p. 2.371). Em maio de 2011, um computador quântico comercial com base em junções Josephson, denominado D-Wave One, concebido para uma aplicação específica, foi anunciado pela empresa D-Wave Systems, dos EUA.
As perspectivas de utilização de pequenos dispositivos supercondutores na eletrônica em geral motivam o estudo da aplicabilidade dos supercondutores de alta temperatura crítica. Nesse caso, a flexibilidade exigida pelos fios não é requerida. No entanto, no caso específico das junções Josephson, a complexidade estrutural e as instabilidades químicas desses materiais comprometem as necessárias garantias de

\section{FIGURA 13}

Arquitetura básica de uma junção Josephson: dois eletrodos supercondutores são separados por uma fina camada de material não supercondutor

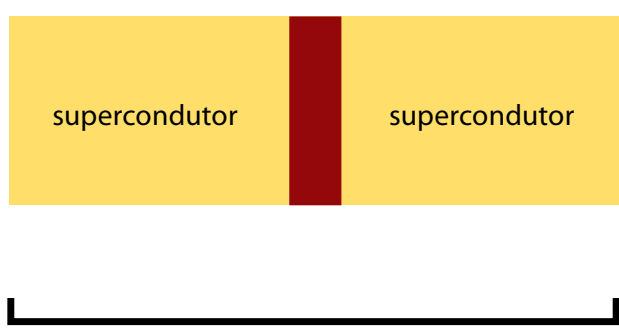

7 Ver artigo de Oswaldo Baffa e Draulio Barros de Araujo, nesta edição. 
confiabilidade e reprodutibilidade na produção de dispositivos em série. Por outro lado, outros pequenos dispositivos, como antenas de ressonadores de micro-ondas, confeccionados com materiais supercondutores de alta temperatura crítica, têm alcançado desempenho muito superior ao obtido com dispositivos realizados com metais nobres. Esse fato tem possibilitado o emprego de tais antenas em estações de radiobase de telefonia celular.

A supercondutividade tem fascinado gerações de cientistas e engenheiros. A manifestação de efeitos quânticos em escala macroscópica, que está na essência do fenômeno, tem desafiado ao longo de décadas a imaginação e engenhosidade dos especialistas na área, muitos dos quais contribuíram com grandes avanços ao conhecimento científico. Dez deles foram laureados com o prêmio Nobel de física, o que, por si só, demonstra a grande importância do tema. A área continua ativa e muitos desafios esperam por superação. Dentre eles estão a des- coberta e caracterização de novos materiais supercondutores de alta temperatura crítica e a proposição de teorias que descrevam adequadamente o estado supercondutor que neles se estabelece. Outros cem anos talvez não sejam suficientes para esgotar os desafios sempre renovados nessa rica e excitante área de pesquisa.

Muitas aplicações tecnológicas dos supercondutores foram concebidas. Provavelmente outras tantas serão inventadas, e é crível que, superadas as dificuldades impostas pelas baixas temperaturas, muitas delas se tornarão parte do dia a dia das pessoas, assim como hoje o são os dispositivos eletrônicos baseados em materiais semicondutores, ou os equipamentos diversos baseados em materiais magnéticos. Nesse caso, esperemos que - muito antes de transcorridos outros cem anos - a tecnologia dos supercondutores esteja amplamente disponível e seja empregada para a melhoria da qualidade de vida da população em geral.

\section{BIBLIOGRAFIA}

BARDEEN, J.; COOPER, L.; SCHRIEFFER, J. R. Physical Review, vol. 106, 1957, p. 162.

BEDNORZ, J. G.; MÜLLER, K. A. Zeitschrift für Physik B, vol. 64, 1986.

HOSONO, H.; REN, Zhi-Na. New Journal of Physics, vol. 11, 025.009, 2009.

DAHL, F. Superconductivity: Its Historical Roots and Development from Mercury to the Ceramic Oxides, American Institute of Physics, 1992.

NAGAMATSU, J.; NAKAYGAWA, N.; MURANAKA, T.; ZENITANI, Y.; AKIMITSU, J. Nature, vol. 410, no 6.824, 2001.

OSTERMANN, Fernanda; PUREUR, Paulo. Temas Atuais da Física. Organizada pela

Sociedade Brasileira de Física. São Paulo, Editora Livraria da Física, 2005.

SHNIRMAN, A.; SCHÖN, G.; HERNON, Z. Physical Review Letters, vol. 79, 1997.

WU, M. K.; ASHBURN, J. R.; TORNG, C. J.; HOR, P. H.; MENG, R. L.; FAO, L.; HUANG, Z. J.;

QANG, Y. Q.; CHU, C. W. Physical Review Letters, vol. 58, 1987. 